\title{
Editorial: Blockchain in Health Care
}

\author{
Nichola Cooper* \\ University of the Sunshine Coast, Maroochydore, QLD, Australia \\ Keywords: blockchain, Health, healthcare, Coronavirus, COVID-19
}

\section{Editorial on the Research Topic}

\section{Blockchain in Healthcare}

As the editors compiled the submissions for this review, the COVID-19 pandemic (the pandemic) swept across the world, endangering and killing millions (Worldometer, 2021) and placing global healthcare systems under unprecedented stress. The impacts of blockchain technologies are commonly discussed in financial and technological domains but never before have the use cases for blockchains in healthcare been so apparent. This review of blockchains used in healthcare settings contains articles addressing COVID-19 use cases, such as managing the systemic effects of the pandemic and containing the spread of the virus.

Blockchain is a form of decentralised ledger technology that enables the secure storage and transmission of data. Each transaction is recorded across each node on the network, meaning that no record can be retroactively altered without altering any subsequent blocks in the chain. Decentralising data recording provides security and stability in data ownership and management and in complex healthcare supply chains, provides financial and logistical traceability (Saberi et al., 2018).

Without a common consensus across the network, blockchain-based records cannot be changed. This feature has lent itself to, most commonly, the consideration of the technology for creating e-health records (Yao et al.) and sharing patient data (Saleh and Shayor), improving hospital administration (Solve Care, 2021), and decreasing hospital costs through more efficient data integration and processing.

While their functional application is nascent, blockchains may assist healthcare systems address fundamental legacy problems. Crucially, for burdened healthcare services, blockchains have a plugand-play type of interoperability that can serve as a backbone for other enabling technologies supporting the introduction of artificial intelligence, $\mathrm{m}$ - and e-health applications and devices and the evolution of the Internet of Medical Things. Introducing a blockchain-enabled environment may support healthcare systems' evolution from beleaguered behemoths to becoming adaptive and responsive.

The inherent transparency, interoperability and security features of blockchains make them uniquely suited to employment within a healthcare environment amidst a global pandemic. Indeed, it may even have taken the COVID-19 pandemic to make governments and health organisations amenable to the value proposition of blockchain technology. Blockchains can verify, secure and share data-useful for confirming identity, vaccination status and permission to leave home (Chamola et al., 2020; IBM, 2020) or prevent health worker fatigue and promote targeted early intervention (Dhillon et al.). They are ideal for secure track and trace, enabling the on-demand design and delivery of custom medical equipment (Medical Parts and Rapid, 2021; Veritx, 2021) and identifying COVID-19 carriers and viral hot-spots. For example, MiPasa, the open data project of the World Health Organisation, IBM, Microsoft, multiple international governments and health organisations, using the HACERA blockchain (Hacera, 2021), plans to detect active COVID-19 cases and share this information with close contacts, hospitals and public health authorities that rely in this information for containment. 
Using blockchain technologies for track and trace efforts also improves citizens' relationship with governments. Contrary to public concern regarding surveillance and privacy when governments have used civilian phone data to track active cases (Kuo, 2020; Tidy, 2020), Civitas is a blockchain initiative of the Honduran government that couples blockchain with geographic information system technologies to connect identities with health records. Each health record contains dates and times of their symptoms providing officials specific, secure, information to support public safety through containment efforts. Citizens may be more likely to trust their governments when governments use secure technologies to store and share their data.

Ultimately, what connects each article in this series, which is one of the core value propositions for the use of blockchain technologies in healthcare, is the ability to provide real-time trustworthy data for analysis, planning and modelling projections. All authors highlight the importance of locating, verifying and sharing user-centric data to aid in treatment and safety-based interventions, prepare for and manage incidents, calibrate state and national responses and promote effective policy-making. Providing secure access to individual data harmonises national responses in pandemic recovery through the collaborative data-sharing ecosystem

\section{REFERENCES}

Chamola, V., Hassija, V., Gupta, V., and Guizani, M. (2020). A Comprehensive Review of the COVID-19 Pandemic and the Role of IoT, Drones, AI, Blockchain, and 5G in Managing its Impact. IEEE Access 8, 90225-90265. doi:10.1109/ACCESS.2020.2992341

Hacera (2021). Intelligent Data and Digital-Asset Exchange - Any Time, Any where. Available at: https://hacera.com/.

IBM (2020). MiPasa Project and IBM Blockchain Team on Open Data Platform to Support COVID-19 Response. Available at: https://www.ibm.com/blogs/ blockchain/2020/03/mipasa-project-and-ibm-blockchain-team-on-open-dataplatform-to-support-covid-19-response/.

Kuo, L. (2020). 'The New normal': China's Excessive Coronavirus Public Monitoring Could Be Here to Stay. Available at: https://www.theguardian.com/world/2020/mar/09/thenew-normal-chinas-excessive-coronavirus-public-monitoring-could-be-here-to-stay.

Medical Parts, Rapid (2021). Rapid Medical Parts; A Trusted Digital Supply Chain. Available at: http://rapidmedicalparts.com/.

Saberi, S., Kouhizadeh, M., Sarkis, J., and Shen, L. (2018). Bockchain Technology and its Relationships to Sustainable Supply Chain Management. Int. J. Prod. Res. 57 (7). doi:10.1080/00207543.2018.1533261

Solve Care (2021). Blockchain Platform for Digital Health Networks. Availableat: https://solve.care/. that blockchain technology can create. In creating permissioned data transparency, varied sectors can supply and predict necessary resources, reducing shortfalls in equipment and vaccines and enabling improved resourcesharing.

The administrative and compliance hurdles to introducing blockchains into highly regulated medical environments are not negated because of a pandemic, however. Dhillon et al. provide an example of how the United States' Health Insurance Portability and Accountability Act 1996 will influence the design of the blockchain architecture and data ownership principles. It should, therefore, not be expected that organisations' and governments' concerns will more quickly embrace blockchain by the global pressure exerted by a novel human virus SARS$\mathrm{CoV}-2$. However, in order to reduce mortality and the economic impact of lockdowns, leaders may consider system efficiencies where possible.

\section{AUTHOR CONTRIBUTIONS}

The author confirms being the sole contributor of this work and has approved it for publication. The draft works were edited prior to publication by Wendy Charles.

Tidy, J. (2020). Coronavirus: Israel Approves Emergency Spy powers. Availableat: https://www.bbc.com/news/technology-51930681.

Veritx (2021). Trusted B2B Marketplace for Industry 4.0. Availableat: https:// veritx.co/.

Worldometer (2021). Coronavirus Cases. Availableat: https://www.worldometers. info/coronavirus/.

Conflict of Interest: The author declares that the research was conducted in the absence of any commercial or financial relationships that could be construed as a potential conflict of interest.

Publisher's Note: All claims expressed in this article are solely those of the authors and do not necessarily represent those of their affiliated organizations, or those of the publisher, the editors and the reviewers. Any product that may be evaluated in this article, or claim that may be made by its manufacturer, is not guaranteed or endorsed by the publisher.

Copyright (C) 2022 Cooper. This is an open-access article distributed under the terms of the Creative Commons Attribution License (CC BY). The use, distribution or reproduction in other forums is permitted, provided the original author(s) and the copyright owner(s) are credited and that the original publication in this journal is cited, in accordance with accepted academic practice. No use, distribution or reproduction is permitted which does not comply with these terms. 